\title{
High Order Aberration Correction in a Post-Column Energy Filter
}

\author{
Nanda K. Menon, Melanie Barfels and Ray Twesten
}

Gatan Inc., 5794 W. Las Positas Blvd., Pleasanton, CA 94588, USA

There have been many improvements in electron optics for the next generation of Gatan Imaging Filters (GIF), collectively known as GIF Quantum ${ }^{\mathrm{TM}}$ [1]. As part of the design, there are 8 postprism dodecapoles, each arranged on a single magnetic yoke and yielding a total of 96 externally controllable lens elements. Robust alignment of the instrument is critical to its performance and is made possible through this improved lens configuration, and also with advances in the algorithms used for automated tuning. As a result, it is now possible to efficiently correct previously ignored higher order aberrations.

Due to the non-round lens optics employed in post-column energy filters, automated aberration correction has been a reality for a number of years. However, the limits imposed by hardware have constrained not just the range of aberrations that may be addressed, but also the methods used for tuning. The number of available lenses in GIF Quantum ${ }^{\mathrm{TM}}$, along their relatively orthogonal effects with respect to the aberrations being addressed, has resulted in a new approach to auto-tuning that involves simultaneous correction using multiple lenses. The lens corrections $\left\{\Delta \mathrm{L}_{i}\right\}$ can be expressed as :

$$
\Delta M_{j}=\sum_{i=1}^{N} \frac{d M_{j}}{d L_{\mathrm{i}}} \Delta L_{i}
$$

For the set of lenses $\{\mathrm{Li}\}$ associated with a given tuning component such as non-isochromaticity, $\Delta \mathrm{L}_{\mathrm{i}}$ is the change required to minimize an aberration $\left(\Delta \mathrm{M}_{\mathrm{j}}=0\right)$. The term $\mathrm{dM}_{\mathrm{j}} / \mathrm{dL}_{\mathrm{i}}$ is referred to as the 'coupling coefficient' and links a given aberration to all possible lenses that might affect it. The above method can be applied to all the tuning routines in both imaging and spectroscopy mode. While spectral tuning is described elsewhere [2], the emphasis here is energy-filtered transmission electron microscopy (EFTEM) mode. Figure 1 shows the effect of $3^{\text {rd }}$ order image distortion correction in GIF Quantum ${ }^{\mathrm{TM}}$ using a group of post-slit quadrupoles. The measurement involves acquisition of a CCD image from a 7x7 mask pattern aperture and characterization of the deviations in observed hole positions from expected values in terms of fitted distortion surfaces. After auto-tuning, the maximum and RMS percentage distortion variation is a factor of 2 lower than what is achieved for the older GIF Tridiem ${ }^{\mathrm{TM}}$ model, which uses only sextupoles.

The GIF Quantum ${ }^{\mathrm{TM}}$ contains a $9 \mathrm{~mm}$ entrance aperture, corresponding to a factor of 3.2 increase in collection area over the Tridiem ${ }^{\mathrm{TM}}$. The larger angular range results in greater EELS signal, but also increases the effects of higher order aberrations on the focusing of the energyloss spectrum at the plane of the energy-selecting slit. This leads to a reduced effective energy resolution. To correct for this, $4^{\text {th }}$ and $5^{\text {th }}$ order isochromaticity auto-tuning is utilized with a combination of pre-slit decapoles and dodecapoles. A typical result is shown in Figure 2, which depicts the final, tuned isochromat after this correction. The maximum non-isochromaticity is 
now less than $2 \mathrm{eV}$, the latter corresponding to the specification for the Tridiem $^{\mathrm{TM}}$ using a $5 \mathrm{~mm}$ entrance aperture.

The removal of chromatic distortions represents another important correction for EFTEM. Figure 3 shows the effect of $3^{\text {rd }}$ order $x$ and y chromaticity tuning performed on GIF Quantum ${ }^{\mathrm{TM}}$. The figure demonstrates the effect of tuning in reducing the differential displacement of mask image holes generated from +/- $25 \mathrm{eV}$ energy shifts applied to the incident electron beam. The high order improvements manifest as the reduced displacement of not just the central mask hole, but also the adjacent surrounding holes, as is demonstrated in the figure. Along with the advances in spectral tuning, the high order image mode auto-tuning is a significant step for ensuring that the GIF Quantum $^{\text {TM }}$ is optimized for both EFTEM and EELS data acquisition.

\section{References}

1. Gubbens et. al., Ultramicroscopy (2010) [in print]

2. Menon et al., Microscopy and Microanalysis, 15(S2), (2009), 212.

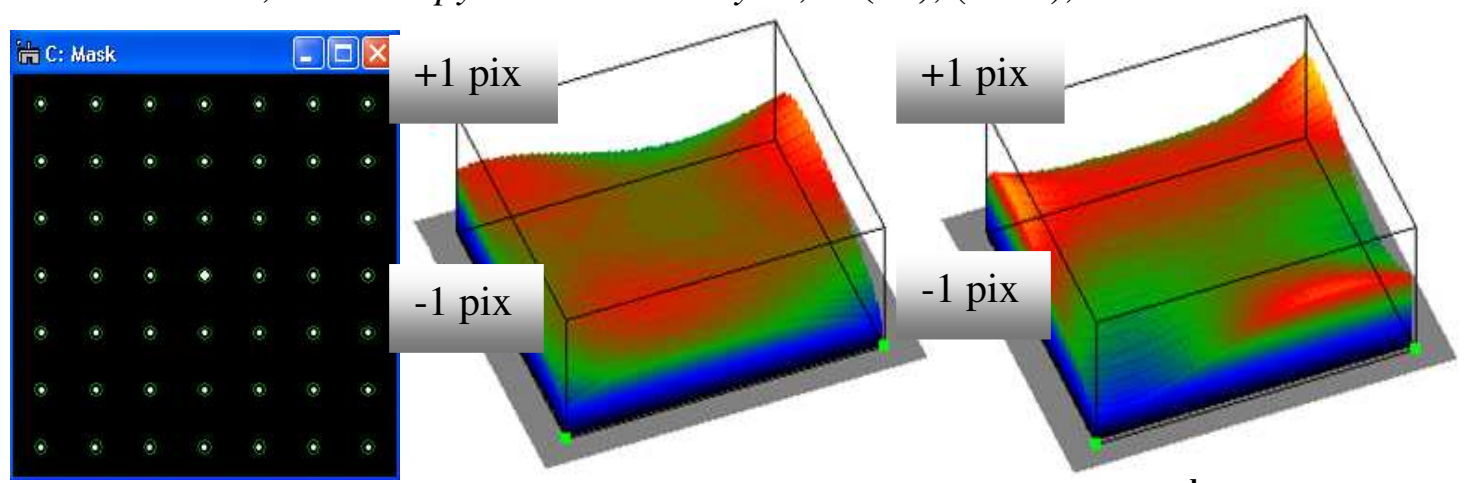

Figure 1. Tuned mask image and $X$ and $Y$ distortion maps after $3^{\text {rd }}$ order correction in GIF Quantum $^{\mathrm{TM}}$. The max. and RMS distortion are $<0.5 \%$ and $0.25 \%$ respectively.

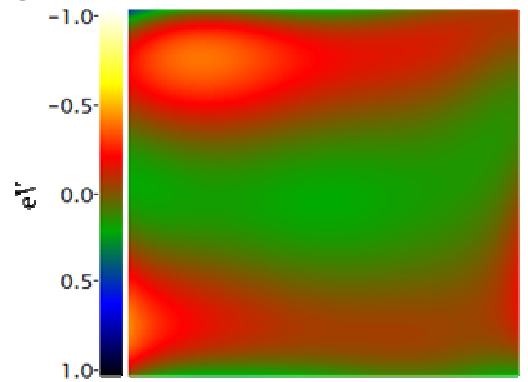

Figure 2. Tuned isochromat after $5^{\text {th }}$ order decapole tuning yielding $1.8 \mathrm{eV}$ max. nonisochromaticity.

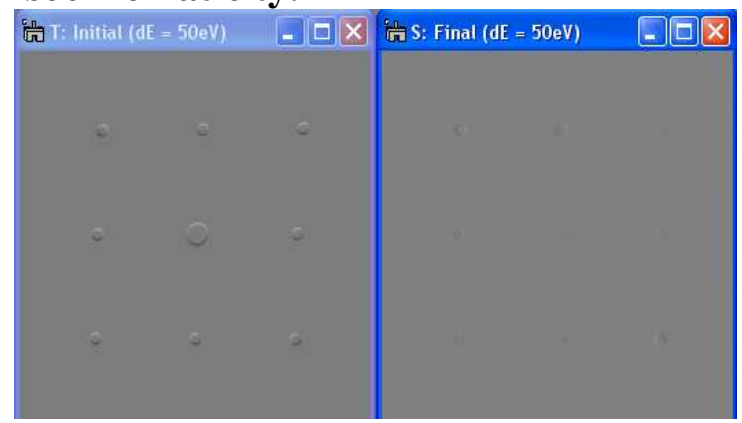

Figure 3. Differential (from application of $+/-25 \mathrm{eV}$ high tension offsets) mask image before and after chromaticity correction. The final max. chromaticity distortion is $0.55 \%$ over $50 \mathrm{eV}$. 